\title{
A Helium White Dwarf of Extremely Low Mass
}

\author{
James Liebert ${ }^{1}$, P. Bergeron ${ }^{2}$, Daniel Eisenstein ${ }^{1}$, H. C. Harris ${ }^{3}$, S. J. Kleinman ${ }^{4}$, Atsuko Nitta ${ }^{4}$, \\ Jurek Krzesinski ${ }^{4,5}$
}

\begin{abstract}
We analyze the spectrum of an unusually-low mass white dwarf, SDSSJ123410.37022802.9 (0335-264-52000), found in our recent, white dwarf catalog from the SDSS DR1 data release. Two independent, model atmosphere fits result in an accurate determination of atmospheric and stellar parameters. The more hands-on analysis yields $T_{\text {eff }}$ $=17,470 \pm 750 \mathrm{~K}$ and $\log g=6.38 \pm 0.05$. We argue that the object cannot be a main sequence A star, a horizontal branch or subdwarf B star. Instead, it is interpreted as a very low mass white dwarf with a core composed of helium, with mass $\left(\sim 0.18-0.19 M_{\odot}\right)$, similar to that published previously for the unusual companion to the millisecond pulsar $\mathrm{J} 1012+5307$. The star probably remains in a binary, perhaps even with an undiscovered or dead pulsar companion. However, the companion might be a more-ordinary star, provided Roche lobe mass transfer began shortly after the now-visible component left the main sequence. A second SDSS low mass white dwarf candidate is also analyzed, but the spectrum for this fainter object is of poorer quality. The sample appears to include additional, similar candidates, worthy of more accurate observation. Correct identification of any additional white dwarfs of extremely low mass requires careful observation and interpretation.
\end{abstract}

Subject headings: white dwarfs - stars: fundamental properties

\section{Introduction}

Kleinman et al. (2004, hereafter K04) have released a catalog of 2561 white dwarf stars found in the first data release of the Sloan Digital Sky Survey (Abazajian et al. 2003, DR1; see also http://www.sdss.org/dr1). As value added to the release, an automatic fitting procedure (autofit)

\footnotetext{
${ }^{1}$ Department of Astronomy and Steward Observatory, University of Arizona, Tucson, AZ 85721

${ }^{2}$ Département de Physique, Université de Montréal, C.P. 6128, Succ. Centre-Ville, Montréal, Québec, Canada, H3C 3J7.

${ }^{3}$ U.S. Naval Observatory, Flagstaff Station, Flagstaff AZ 86002-1149

${ }^{4}$ Apache Point Observatory, P.O. Box 59, Sunspot NM 88349-0059

${ }^{5}$ Mt. Suhora Observatory, Cracow Pedagogical University, ul. Podchorazych 2, 30-084 Cracow, Poland
} 
was used to estimate the parameters $T_{\text {eff }}$ and $\log g$ for each white dwarf classified DA and DB. Extensive human checks of the "outliers" in the $T_{\text {eff }}$ vs $\log g$ plot resulted in elimination of many invalid fits, due usually to a corrupted spectrum or flux calibration. However, K04 noted several white dwarfs of unusually low surface gravity which appear to be valid, if usually inaccurate, fits.

While it has been known for a long time that the mass distribution of field DA white dwarfs peaks near $0.6 M_{\odot}$ (Koester, Schulz, \& Weidemann 1980), the more precise determinations of $T_{\text {eff }}$ and $\log g$ made possible by accurate digital spectra and model atmosphere fits to the Balmer lines demonstrated the reality of both separate high mass and low mass components to this mass distribution (Bergeron, Saffer, \& Liebert 1992; Bragaglia, Renzini, \& Bergeron 1995). With derived masses $\lesssim 0.5 M_{\odot}$, the latter were inferred to have helium cores, requiring envelope mass loss to a companion before the progenitor reached the tip of the red giant branch and could ignite the helium. This prediction was dramatically confirmed by Marsh, Dhillon, \& Duck (1995) and Maxted \& Marsh (1999), who found that a large fraction of these stars and other low gravity DA white dwarfs were indeed binaries with orbital periods of hours to days. The inferred companion was generally another white dwarf, sometimes detectable in the spectrum. In a few cases, an infrared excess was discovered, which implied a low mass nondegenerate companion (Zuckerman \& Becklin 1992).

The apparent helium white dwarfs in the Bergeron et al. (1992) and Bragaglia et al. (1995) samples of stars with $T_{\text {eff }}<50,000 \mathrm{~K}$ had $\log g$ as low as 7.22 and masses as low as $0.31 M_{\odot}$ (PG 1241-010). Very hot, low mass white dwarfs have radii much larger than the final "zerotemperature" values. For instance, in Bergeron et al.'s (1994) analysis of hot DAO stars, the lowest gravity fitted was for the star HZ34 at $\log g=6.61$ and $T_{\text {eff }}=60,700 \mathrm{~K}$. Probably the lowest mass white dwarf reported up to now has a most unusual evolutionary history. It is the companion to the millisecond pulsar J1012+5307. Presumably it spun-up the pulsar during the prior common envelope phase. Van Kerkwijk, Bergeron, \& Kulkarni (1996) found for the white dwarf $T_{\text {eff }}=$ $8,550 \pm 25 \mathrm{~K}$ and $\log g=6.75 \pm 0.07$, from which they estimated a tiny mass of only $0.16 \pm 0.02 M_{\odot}$. Callanan, Garnavich, \& Koester (1998) derived $T_{\text {eff }}=8670 \mathrm{~K}, \log g=6.34$, but the same mass of $0.16 \pm 0.02 M_{\odot}$ in an independent analysis.

Several stars in the K04 DA sample fit surface gravities lower than those cited above. For most of these mentioned in K04, Section 7.1, the spectrophotometry of these stars near 20th magnitude is too poor for accurate determinations of parameters. Improved spectra would be recommended. For convenience, the SDSS photometry for the stars mentioned in the cited paper is listed in Table 1.

The main purpose of this paper is to develop the case for an extremely low mass for the bestobserved example, SDSSJ 123410.37-022802.9 (hereafter, SDSSJ1234), a result in which we have high confidence. We do include analysis of one other low mass candidate, to illustrate what happens with a poorly-detected spectrum. In Section 2, independent fits to the SDSS spectrophotometry of the Balmer lines are presented. In Section 3, we discuss the implications. 


\section{Determination of parameters}

SDSSJ1234 is a fairly bright star compared to most cataloged in K04, at $\mathrm{g}=17.87$. The proper motion measurement $\mu=13$ milliarcseconds per year is probably real. The "autofit" techniques

are discussed in the cited paper. For this star the fitted $T_{\text {eff }}=17,500 \mathrm{~K}$, with $\log g=6.375$. The fit is shown in Figure 13 of K04.

Extraordinary claims, however, should be backed up by careful analysis. We therefore performed a careful, hands-on fit to the spectrum using the independent pure hydrogen models of one of us (PB). The method of analysis is that discussed in the previously-cited Bergeron et al. (1992), Bragaglia et al. (1995), and many other Bergeron papers. The fit is shown in Figure 1(left). The resulting parameters are $T_{\text {eff }}=17,470 \mathrm{~K} \pm 750, \log g=6.38 \pm 0.05$, in excellent agreement with the autofit values in K04. We can thus be confident that these are good estimates of the parameters of this unusual star.

It is possible that other DA white dwarfs of unusually-low mass are included in the DR1 Catalog. Several are mentioned in K04. The problem of analyzing a 2.5-meter spectrum of a much fainter candidate is illustrated in Figure 1(right), an attempt to fit the Balmer lines of SDSSJ 105611.03+653631.5 $(\mathrm{g}=19.77)$ with Bergeron models. The derived parameters are $T_{\text {eff }}=$ $21,910 \pm 1900 \mathrm{~K}, \log g=7.07 \pm 0.09$. The autofit in the catalog gives $18,149 \pm 288 \mathrm{~K}$ and $6.73 \pm 0.10$, respectively. Reobservation of this star to achieve a high signal-to-noise ratio is desirable to determine more accurate parameters.

We do not judge it to be worthwhile to show fits to the remaining low mass candidates in the paper cited above. For instance, the very noisy spectrum of SDSSJ234536.48-010204.8 - which is discussed more extensively in K04 - fit parameters of $T_{\text {eff }}=27,141 \mathrm{~K}, \log g=6.06$ with the autofit package. With Bergeron models and techniques, the parameters are considerably different at $34,042 \mathrm{~K}$ and 6.77 . Clearly this is an example of a candidate which needs much more precise observation.

\section{The Nature of SDSSJ1234}

We note that the SDSS colors of SDSSJ1234, $u-g=+0.32, g-r=-0.35$, are similar to DA or subdwarf B stars of similar $T_{\text {eff }}$. Actually, these accurate colors place the object to the right of the Bergeron $\log g=7$ curve for hydrogen-atmosphere white dwarfs plotted in the $u-g-g-r$ diagram (Figure 1) in the Harris et al. (2003) paper. This paper presented the first sampling of white dwarfs from the SDSS. The colors thus are in agreement with the determination that SDSSJ1234 has $\log g$ less than 7 .

Clearly, these colors are inconsistent with main sequence A or horizontal branch A stars which have somewhat similar $\mathrm{H}$ line profiles, but much larger Balmer jumps $(u-g \sim 1)$. The fitted gravity is also higher than for these types of stars, although this may not be obvious from simple inspection 
of the hydrogen line spectrum.

Could SDSSJ1234 be a subdwarf B (sdB) star on the extended horizontal branch? Such stars can have similar colors. However, this interpretation cannot be correct. Saffer et al. (1994) analyzed a sample of bright, field sdB stars using a similar analysis of Balmer line spectra at these lower surface gravities. In their Figure 5, the distribution is plotted in $\log g$ vs. $T_{\text {eff }}$. Along this EHB, the $\log g$ increases with increasing Teff, but the upper limit to $\log g$ at a given Teff is believed to be specified by models for the "zero age" horizontal branch (HB), the location of the star at the start of the core-helium burning phase. Stars evolve upwards in luminosity to lower log $g$ values as the He-burning proceeds. HB models were also plotted in the Saffer et al. figure. It is shown that a star of $17,000 \mathrm{~K}$ is expected to have $\log g$ no higher than 5.0, lower by more than an order of magnitude.

If the proper motion is real, the implied tangential velocity of the star at the required absolute magnitude and distance for the horizontal branch might leave it unbound to the galaxy. At $\mathrm{M}_{V} \sim+2, \mathrm{~g}=17.88$ is roughly $\mathrm{V}=17.98$, then $\mathrm{d}=15,700 \mathrm{pc}$. Thus $\mathrm{v}_{\text {tan }}=4.738 \mu \mathrm{d} \sim 970 \mathrm{~km} \mathrm{~s}^{-1}$. If instead we use the fitted $\mathrm{M}_{V}=+8.22, \mathrm{v}_{\tan }=55 \mathrm{~km} \mathrm{~s}^{-1}$, appropriate for the old or thick disk population at a distance of about $900 \mathrm{pc}$.

The star lies in luminosity between the sdB/HB sequence and the sequence of normal-mass white dwarfs. At $\mathrm{M}_{V}=+8.22$, it is nearly six magnitudes fainter than the $\mathrm{HB}$ at $17,000 \mathrm{~K}$, and 2.7 magnitudes brighter than a $17,000 \mathrm{~K}$ white dwarf with mass $0.6 M_{\odot}$. The only possible interpretation appears to be that it is a helium white dwarf with unusually low mass and large radius. The estimated radius is $0.047 R_{\odot}$, about four times that of a $0.6 M_{\odot}$ white dwarf. At an estimated $\mathrm{M}_{b o l}=+6.57$, it is about one fifth of the luminosity of the Sun!

How extreme are the parameters? In Figure 2, we plot the six evolutionary sequences of Althaus et al. (2001), and Serenelli et al. (2001) in a temperature - gravity diagram, along with the positions of the two SDSS stars analyzed in the previous section, and the white dwarf companion to the millisecond pulsar discussed in the introduction. SDSSJ1234 appears to fit a mass not significantly different from $0.196 M_{\odot}$, and essentially the same as these evolutionary sequences would estimate for the pulsar companion. However, these values also depend on whether gravitational diffusion and a pure-hydrogen atmospheric composition are correct assumptions, and on the assumed thickness of the hydrogen envelope. Note that Althaus et al. (2001) estimated $0.169 M_{\odot}$ for the pulsar, using the lower gravity of Callanan et al. (1998). The values for these two unusual white dwarfs obviously remain uncertain. Finally, however, we note the even the mass inferred for SDSSJ $105611.03+653631.5$ is near the $0.31 M_{\odot}$ value for the lowest mass white dwarf previously determined in the literature (see the Introduction), apart from the pulsar companion.

It is very likely that SDSSJ1234 has a binary companion, as apparently do the other low mass, helium-core white dwarfs. If unusual evolution in a common envelope with a neutron star is required for so much of the original hydrogen envelope to be removed, then we would infer that this white dwarf could have an undiscovered or dead pulsar companion. 
If the binary separation is appropriate, however, it is possible that the companion might just be a cool white dwarf following more ordinary common envelope (CE) evolution. For instance, if the progenitor of the observed component were $1 M_{\odot}$, it leaves the main sequence and reaches the so-called Schönberg-Chandrasekhar limit with a helium core of about $0.1 M_{\odot}$ (see, e.g. Kippenhahn \& Weigert 1990, p. 285). The radius slowly expands from this point and the companion must be located such that the evolving subgiant overfills its Roche lobe soon afterwards, and begins unstable mass transfer to the degenerate companion. If the subsequent CE phase is sufficiently unstable, most of the envelope mass may be lost quickly from the system, so that the core mass of the evolving star does not grow past $0.16-0.19 M_{\odot}$. Thus, the phenomenon of ultra-low mass He white dwarfs may occur in the ordinary field population from more-ordinary binary star evolution.

We conclude by emphasizing that the correct identification of any extremely low mass white dwarfs like SDSSJ1234 in various surveys requires careful observation and interpretation. In the past, some might have been dismissed by inspection of the spectra as some other class of star than a white dwarf. The SDSS will be an ideal sample for a robust determination of frequency of such objects.

This work was supported by the National Science Foundation through grant AST-0307321 for study of white dwarfs found in the SDSS. This work was also supported in part by the NSERC Canada and by the Fund NATEQ (Québec). 


\section{REFERENCES}

Abazajian, K. et al. 2003, AJ, 126, 2081

Althaus, L.G., Serenelli, A.M., Benvenuto, O.G. 2001, MNRAS, 323, 471

Bergeron, P., Saffer, R.A., \& Liebert, J. 1992, ApJ, 394, 228

Bergeron, P., Wesemael, F., Beauchamp, A., Wood, M.A., Lamontagne, R., Fontaine, G., Liebert, J. 1994, ApJ, 432, 305

Bragaglia, A., Renzini, A., \& Bergeron, P. 1995, ApJ, 443, 735

Callanan, P.J., Garnavich, P.M., \& Koester, D. 1998, MNRAS, 298, 207

Harris, H. et al. 2003, AJ, 126, 1023

Kippenhahn, R., \& Weigert, A. 1990, Stellar Structure and Evolution, Astronomy and Astrophysics Library, Springer-Verlag: Berlin

Kleinman, S.J., et al. 2004, ApJ, 607427 (K04)

Koester, D., Schulz, H., \& Weidemann, V. 1980, A\&A, 76, 262

Marsh, T.R., Dhillon, V.S., Duck, S.R. 1995, MNRAS, 275, 828

Maxted, P.F.L., \& Marsh, T.R. 1998, MNRAS, 296, L34

Saffer, R.A., Bergeron, P., Koester, D., \& Liebert, J. 1994, ApJ, 432, 351

Serenelli, A.M., Althaus, L.G., Rohrmann, R.D., \& Benvenuto, O.G. 2001, MNRAS, 325, 607

Van Kerkwijk, M.H., Bergeron, P., \& Kulkarni, S.R. 1996, ApJ, 467, L89

Zuckerman, B., \& Becklin, E.E. 1992, ApJ, 386, 260 
Fig. 1.- The simultaneous fits to the normalized Balmer lines of SDSSJ1234 (left frame), and SDSSJ105611.03+653631.5 (right frame), using line profiles calculated from Bergeron models. This technique is described in Bergeron papers given in the text.

Fig. 2. - The positions of the two SDSS stars analyzed here, and the white dwarf companion to the millisecond pulsar J1012+5307, in a $T_{\text {eff }}$ vs. $\log g$ diagram, using the Bergeron fits. Van Kerkwijk et al. (1996) parameters are adopted here for the pulsar companion. Several evolutionary tracks for very low mass helium core models from Althaus et al. (2001) and Serenelli et al. (2001) are shown for comparison.

Table 1. SDSS Photometry of Helium White Dwarf Candidates

\begin{tabular}{lcrrrrrrrrr}
\hline \hline \multicolumn{1}{c}{ SDSSJ } & $\mathrm{g}$ & $\mathrm{u}-\mathrm{g}$ & $\mathrm{g}-\mathrm{r}$ & $\mathrm{r}-\mathrm{i}$ & $\mathrm{i}-\mathrm{z}$ & $\sigma_{u}$ & $\sigma_{g}$ & \multicolumn{1}{c}{$\sigma_{r}$} & $\sigma_{i}$ & $\sigma_{z}$ \\
\hline $002207.65-101423.5$ & 19.77 & -0.11 & -0.30 & -0.19 & -0.29 & 0.05 & 0.02 & 0.03 & 0.04 & 0.22 \\
$081136.34+461156.4$ & 19.76 & -0.17 & -0.44 & -0.40 & 0.02 & 0.03 & 0.02 & 0.03 & 0.05 & 0.17 \\
$102228.02+020035.2$ & 20.15 & 0.20 & -0.30 & -0.36 & -0.13 & 0.05 & 0.04 & 0.04 & 0.07 & 0.28 \\
$105611.03+653631.5$ & 19.77 & 0.15 & -0.39 & -0.24 & -0.17 & 0.05 & 0.03 & 0.03 & 0.05 & 0.19 \\
$123410.37-022802.9$ & 17.88 & 0.33 & -0.37 & -0.21 & -0.25 & 0.09 & 0.02 & 0.02 & 0.03 & 0.06 \\
$130422.65+012214.2$ & 19.33 & 0.45 & 0.19 & -0.02 & 0.04 & 0.04 & 0.03 & 0.02 & 0.02 & 0.06 \\
$142601.48+010000.2$ & 19.35 & 0.31 & -0.33 & -0.24 & -0.22 & 0.04 & 0.02 & 0.03 & 0.04 & 0.12 \\
$234536.48-010204.8$ & 19.50 & -0.28 & -0.42 & -0.28 & -0.32 & 0.03 & 0.02 & 0.02 & 0.04 & 0.16 \\
\hline
\end{tabular}




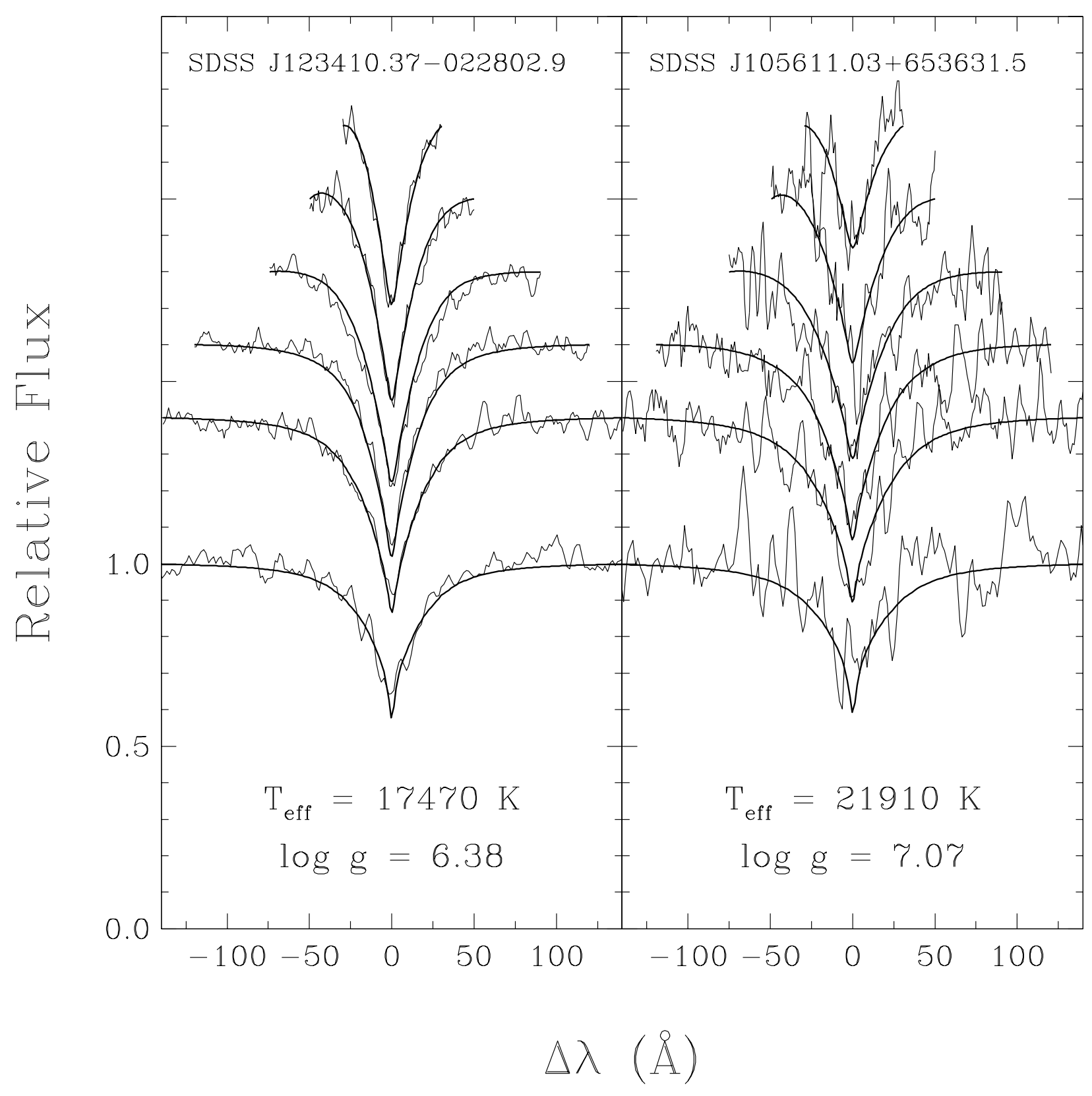




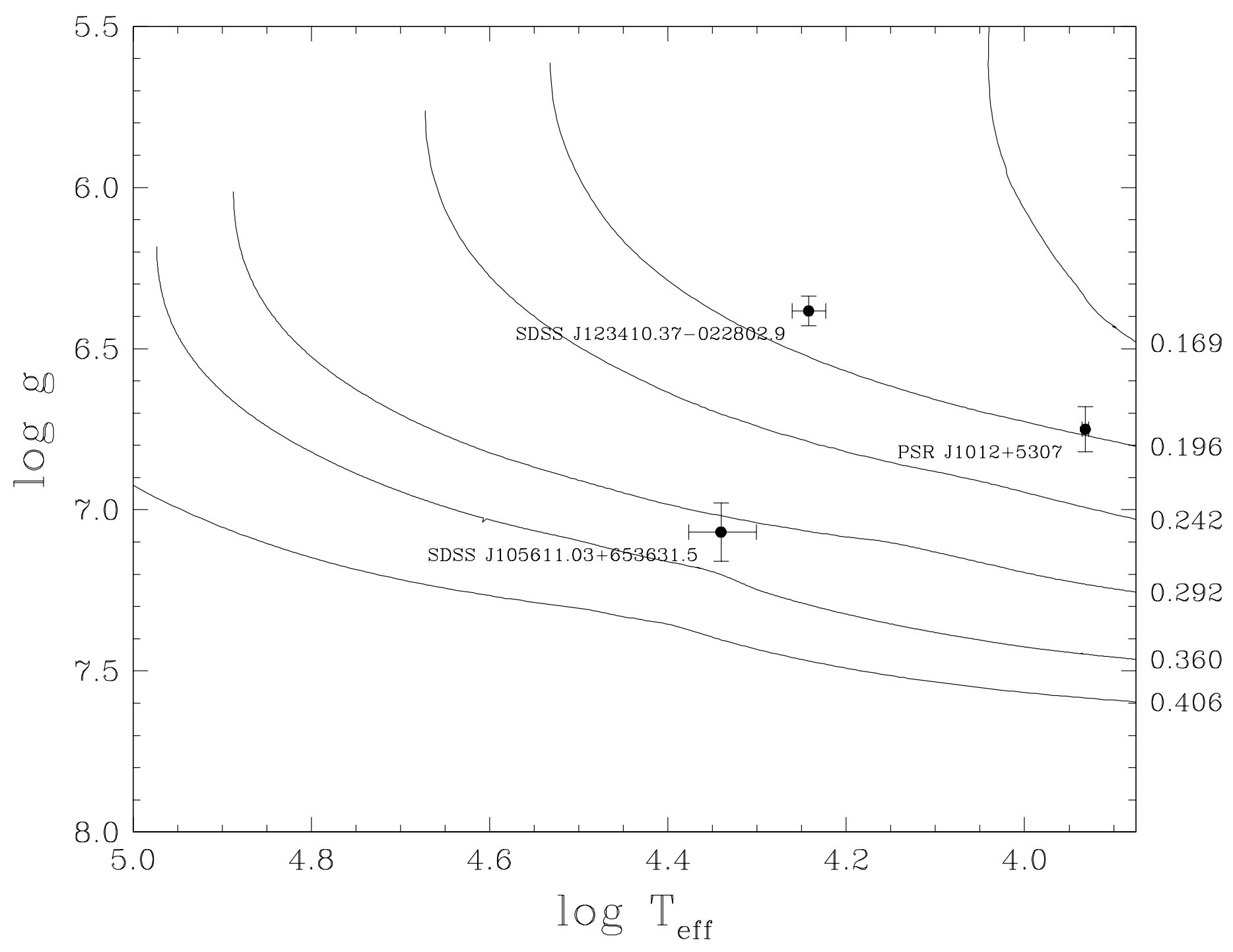

